\title{
STRATÉGIES D'ACTION POUR UNE RECONSTRUCTION DE L'IDENTITÉ DE GENRE DE LA FEMME RURALE MAROCAINE
}

\section{ACTIONS STRATEGIES FOR THE RECONSTRUCTION OF GENDER IDENTITY AMONG MOROCCAN RURAL WOMEN}

\section{Laila Hilal}

Université Mohamed V, Rabat. Marruecos/Morocco hilal laila@yahoo.fr

"On ne naît pas femme, on le devient" S. de Beauvoir, Le Deuxième Sexe, 1949.

Recibido/Received: 14/05/08

Aceptado/Accepted: 07/07/08

\section{RÉSUMÉ}

A travers cette contribution, fruit d'un travail de recherche plus large sur l'identité de la femme (construction et reconstruction) rurale marocaine actuelle, nous tentons une approche des stratégies d'action possibles qui puissent aider la femme à développer des habiletés professionnelles et personnelles qui lui permettent une plus grande participation dans la vie sociale, politique et économique, d'améliorer sa qualité de vie tout en contribuant à l'aider à la reconstruction de son identité féminine dans un esprit de rapport plus égalitaire entre les genres, jusqu'ici en faveur de l'homme.

\section{MOTS CLES}

Identité de genre, femme rurale, construction/reconstruction de l'identité, stratégies d'action.

\section{ABSTRACT}

With through this contribution, fruit of a broader research task on the identity of the current Moroccan rural woman, we try an approach of the possible strategies of action which can help the woman to develop skills professional and personal which allow him a greater participation in the social, political and economic life, to improve its quality of life while contributing to help them with the rebuilding of their female identity in a spirit of more levelling relationship between the kinds, up to now in favour of the man.

\section{KEYWORDS}

Gender identity, rural woman, construction/reconstruction of the identity, actions strategies. 


\section{INTRODUCTION}

Il ne s'agit pas pour nous dans cette réflexion en quête de la reconstruction de l'identité de genre de la femme rurale marocaine actuelle, prétendre apporter des solutions sous forme d'une série d'actions qui permettraient de répondre à la question que pose cet article: qu'elles stratégies à mettre en œuvre pour une reconstruction de l'identité de la femme rurale marocaine. La question est simple, mais la réponse est complexe et multidimensionnelle. C'est dans l'analyse des problèmes auxquels est confrontée la femme rurale que nous pourrons mieux dégager des stratégies d'action. Par conséquent, c'est dans cette direction que nous orienterons nos efforts dans notre réflexion.

Pour commencer, les études que nous avons mené nous permettent d'avancer l'idée selon laquelle un processus de développement coopératif en milieu rural (ou dans des situations où des femmes vivent la précarité) peut contribuer à la participation des femmes au développement dans le but d'améliorer leur situation sociale et certainement à moyen et à court terme, bouleverser les rapports inégaux entre les genres pour atteindre l'égalité des genres. La transformation de ces rapports contribueraient grandement à l'élimination de la subordination à laquelle est confinée la femme et aussi permettraient à celle-ci de retrouver son identité de femme porteuse d'un potentiel de transformation de son environnement social, économique et politique.

La question est de savoir s'il existe un modèle qui mettrait à jour de nouveaux concepts et les relations qui existeraient entre eux. Il est difficile pour nous de répondre à cette question car nous croyons qu'il est moins urgent d'avancer de nouveaux concepts que de préciser les éléments et les activités essentielles qui doivent accompagner un processus de développement axé sur le genre. C'est-à-dire un projet de développement qui mette :

- En place une entreprise féminine collective (ou même mixte, car il ne s'agit pas d'enfermer la femme dans une structure qui perpétuerait un découpage social injuste et ainsi, maintiendrait des comportements discriminants à son égard).

- En valeur des savoir-faire et des ressources locales, sur lesquelles les femmes ont un certain contrôle et qui fonctionne de façon autonome et indépendamment de l'Etat ou tout autre organisme.

C'est-à-dire, qu'elles aient un espace de travail où elles puissent avoir la possibilité de développer des habiletés professionnelles et personnelles leur permettant d'atteindre un niveau de pleine participation, améliorant ainsi leur niveau de vie et en contribuant à les aider à retrouver leur identité féminine dans les rapports entre les genres jusque-là inégalitaire.

Notre expérience tirée du travail associatif longue de plusieurs années, et nos 
observations sur le terrain nous confortent dans l'idée selon laquelle le modèle coopératif est un modèle porteur d'un fort potentiel de transformation des rapports entre les genres. Ce modèle s'appuie sur trois grands piliers :

a) Un environnement externe de la coopérative qui participera essentiellement au financement (démarrage de l'activité) et un appui technique et logistique.

b) Un environnement interne lui permettant de fonctionner de façon autonome (pleine participation).

c) La capacité de se prendre en charge et de décider de son avenir (empowerment).

De plus, nous considérons que les actions prioritaires tendant à faire émerger un tel modèle de développement coopératif doivent aller dans le sens du renforcement:

- Des capacités financières rendant l'organisation viable financièrement (durabilité économique) et pérenne socialement (durabilité sociale).

- Du processus d'empowerment qui permete aux femmes d'avoir un pouvoir et de travailler à leurs intérêts stratégiques en plus de leurs besoins pratiques, de l'intérieur comme de l'extérieur de leur organisation (durabilité politique).

Ce sont ces deux aspects qui nous paraissent essentiels pour la viabilité de tout projet de développement coopératif durable. Dans ce qui suit nous tenterons de porter une attention particulière à ces deux aspects.

D'autre part, nous pensons que c'est à travers l'analyse des principaux obstacles (limites) à la production et à la participation sociale des femmes (comprendre les raisons d'un "processus de féminisation de la pauvreté ») que l'on trouvera les connaissances nécessaires (voir des réponses) permettant de développer des stratégies d'actions adéquates à la resocialisation et à la reconstruction de leur identité.

En effet, les différentes manifestations et conférences mondiales organisées autour du thème de la femme rappellent sans cesse « la persistante et croissante féminisation de la pauvreté ». C'est un des plus graves problèmes pour atteindre les objectifs d'égalité et de développement entre les genres et accroître la pleine participation de la femme en général et, de la femme rurale en particulier, dans tous les domaines de la société.

Le combat pour un changement de cette situation passe donc nécessairement par un développement durable axé sur le genre.

\section{VERS UN DEVELOPPEMENT AXE SUR LE GENRE}

La problématique de subordination vécue par les femmes constitue le point central autour duquel se forment des partenariats entre organisations locales et 
internationales de développement. A travers ces partenariats, les organisations travaillent à la création de projets de développement durable avec les femmes.

Le raisonnement qui nous conduit à introduire la perspective de genre dans les politiques de développement se base essentiellement sur deux arguments :

Le premier est de nature politico étique. La discrimination par le sexe est une violation des Droits Humains Universels et, par conséquent, une situation qu'il faut dépasser. Depuis la Quatrième Conférence Mondiale sur la Femme, (Beijing, 1995), l'abolition de la discrimination de la femme s'est convertie en un objectif des politiques de développement.

Le deuxième argument est de nature économique. La discrimination de la femme et une politique de développement insensible au genre impliquent des coûts d'efficience, de bien-être et d'équité pour la société (Durstewitz, 2000, La perspective de genre dans les micros finances). Dans beaucoup de cas, la discrimination par sexe produit la pauvreté et freine le développement social et économique. Par conséquent, une plus grande équité et égalité des droits entre hommes et femmes peuvent être considérées comme une condition préalable pour un développement durable.

Souvent, on pense qu'une différenciation de genre est inutile et inécessaire. La famille est perçue alors comme une association «altruiste» dont tous les membres se bénéficient pareillement de tout ce qui concerne le foyer en général. De plus, on suppose que tous les membres d'un foyer ont pareil accès et contrôle sur les ressources. Cette hypothèse est très peu vérifiée car les possibilités de pouvoir bénéficier des ressources dont dispose les membres du foyer, en général, diffèrent avec le genre et l'âge.

C'est pourquoi, Osterhaus S. constate que « ce type d'affirmations fausses est une des causes fondamentales de l'inexistence de conceptions appropriées de projets dont les conséquences sont souvent l'échec ou le faible impact d'un projet de développement ». Pour éviter ces conséquences négatives et inattendues, il est nécessaire d'introduire explicitement une perspective de genre dans les politiques de développement. De plus, des différents rôles que la société assigne aux femmes et aux hommes en découlent aussi des besoins différents.

\section{ERADIQUER LA FEMINISATION DE LA PAUVRETÉ}

Les normes sociales et culturelles en relation au genre ont accentué les désavantages et les limites imposées aux femmes pour une plus grande intégration économique et sociale. L'ensemble des stéréotypes qui font partie de ces évaluations 
sociales et culturelles s'est matérialisé dans des programmes et stratégies d'actions qui tendent à marginaliser les femmes et à les confiner dans des situations discriminatoires. Ces concepts se traduisent en programmes, projets et stratégies orientées vers l'homme, considéré comme le chef de famille et où la femme assume un rôle de dépendance, exerçant des tâches orientées vers des fonctions reproductives du foyer.

Ci-après quelques uns des principaux stéréotypes qui font partie de ces normes sociales et culturelles :

- Les fonctions fondamentales de la femme sont la reproduction et les responsabilités domestiques.

- La femme assume un rôle secondaire dans la génération des revenus du foyer.

- Le travail de la femme ne peut être considéré comme égal à celui des hommes, en termes d'évaluation des apports.

Cette situation a conduit souvent les femmes à:

1 Posséder des niveaux d'éducation et de formation inférieurs à celui des hommes.

$2 \mathrm{Ne}$ pas être prises suffisamment en compte dans la définition des programmes et plans de développement.

3 Avoir un accès limité aux ressources productives (terre, capital, technologie, ...).

4 Connaître des difficultés lors des embauches avec des salaires inférieurs à celui des hommes.

5 Avoir des journées de travail plus longues que celle des hommes.

6 Développer des activités à plus faible rentabilité.

7 Avoir un accès limité au crédit et/ou micro crédit.

8 Disposée d'une expérience limitée dans les domaines entrepreunarial, organisationnel et financier.

9 Dépendre de l'homme en matière de garanties, commercialisation, gestion de l'épargne,...

La Plateforme d'Action adoptée à Beijing et les Objectifs du Millénaire, et en particulier les objectifs 1 et 3, respectivement : «Réduire l'extrême pauvreté et la faim » (dont les femmes et les enfants en sont les principales victimes) et «Promouvoir l'égalité des sexes et l'autonomisation des femmes », ont établi les principaux objectifs stratégiques pour agir sur ce processus :

- Revoir, adopter et entretenir des politiques macros économiques et des stratégies de développement qui prennent en compte les besoins des femmes et appuient leurs efforts pour se soustraire à la pauvreté dans le cadre de processus de développement durable. 
- Revoir les lois et les pratiques administratives pour assurer à la femme une égalité des droits et d'accès aux ressources économiques.

- Donner accès à la femme aux mécanismes et institutions d'épargne et de crédit.

- Entreprendre des recherches pour « utiller » les femmes pour lui permettre de « sortir de la pauvreté ».

Ce processus de féminisation de la pauvreté trouve ses racines historiques dans les processus socio économiques des pays en développement, dans les effets des politiques macros économiques, dans l'évolution même des approches sur le genre et le développement, et dans les règles sociales et culturelles traditionnellement intériorisées.

Dans le passé, les politiques macros économiques se voulaient neutres en relation avec le genre. Les pays en voie de développement tels que le Maroc ont inclus dans leurs analyses des indicateurs classiques de type économique comme par exemple, balance commerciale et de paiements, déficit fiscal, croissance économique, ... La non prise en compte d'indicateurs de bien être humain a conduit à une série de limitations quant aux effets de ces politiques dans une perspective de genre.

D'autre part, on connaît les effets sur la femme des divers Programmes d'Ajustements Structurels (connus sous le sigle PAS), et ce depuis 1982. Les PAS ont encouragé la production de cultures commerciales non traditionnelles et la production à grande échelle pour l'exportation, ce qui a eu comme conséquence d'étouffer la production traditionnelle spécialement de subsistance où la femme jouait un grand rôle. La conséquence de cette politique a été une série de restriction budgétaire dans les dépenses publiques relatives aux subventions dédiées à l'agriculture, l'assistance technique, la commercialisation et les crédits, avantages qu'on a transféré au secteur des cultures non traditionnelles « exportables ». La production à grande échelle a entrainé un surenchérichessement des terres et de leur valeur, provoquant la marginalisation de la femme agriculteur et son confinement sur des terres à faible valeur agricole.

Les PAS ont également conduit à la réduction des dépenses publiques relatives aux services sociaux comme l'éducation, la santé et les infrastructures sociales. Ces programmes contribuèrent à accroître les différences entre les secteurs économiques et à mettre en évidence une nouvelle forme de pauvreté. Cette situation poussa la femme à une plus grande participation dans les secteurs informels et dans les activités à faible rémunération, cherchant à compenser la diminution des revenus familiaux. Ceci a eu des coûts sociaux élevés particulièrement dans les champs de la santé, l'éducation et l'enfance.

Les effets du processus de globalisation et l'ouverture commerciale se font à peine sentir. Certains facteurs comme la coopération décentralisée, une plus grande offre de crédits et de micro crédits, un «associationnisme » accru et une politique publique 
plus sociale ont stimulé la participation de la femme dans l'économie particulièrement en matière d'auto emploi, entraînant ainsi une prolifération de micro entreprises gérées par des femmes.

Dans le milieu rural, où la production agricole exige plus que jamais une plus grande spécialisation et une technicité accrue pour réduire les coûts et pouvoir supporter la concurrence en qualité et en prix, la femme rurale reste encore absente du processus de formation technique et avec un réel handicap face à l'accès aux crédits pour pouvoir entreprendre, au-delà d'une vision de micro crédits.

Parallèlement aux modèles de développement économique, il existe diverses approches relatives au genre et à l'analyse de la pauvreté des 40 dernières années, et partantes, l'implémentation de stratégies d'actions tendant à trouver une réponse en harmonie avec ses spécificités et ses objectifs. De sorte que, la nature et la manière d'aborder ces approches ont eu d'une manière ou d'une autre une incidence sur les processus de féminisation de la pauvreté :

- Approche Assistance sociale ou «Bien être »: Le modèle de développement des années 50 s'est caractérisé par une approche des problèmes des femmes, basée sur son rôle reproductif. La femme est alors considérée comme dépendante de l'homme et sa fonction propre est celle de mère et de femme au foyer. Ce faisant, les politiques, stratégies et messages étaient orientées vers un renforcement de cette vision où la femme participe peu ou pas du tout au système productif e,t de toute façon, ses revenus font partie intégrante des ressources familiales sans aucune distinction.

- Approche Equité: A la fin des années soixante et début soixante dix, l'échec des politiques économiques met à nu les séquelles du retard social et économique accumulé par des femmes. Timidement on commence alors à reconnaître l'apport de la femme au développement, à revoir les relations qui régissent les foyers et à définir des stratégies et des actions correctrices, destinées à réduire le fossé qui sépare les hommes des femmes.

- Approche Anti-pauvreté: À partir des années soixante dix, une meilleure connaissance du phénomène de la pauvreté permet de reconnaître les conditions particulières qui touchent les femmes pauvres et le rôle qu'elles jouent comme pilier du foyer. Les stratégies adoptées permirent de créer des opportunités d'emploi pour les femmes en cherchant à leur donner un rôle plus actif et à rendre visible leurs apports à la société en général et à l'économie en particulier.

- Approche Efficience: Cette approche fit son apparition au début des années quatre vingt, et considéra que les opportunités de développement d'un pays étaient conditionnées par la pleine utilisation des ressources humaines disponibles, d'où 
l'intégration de la femme à la société et à l'économie devient fondamentale pour le développement. De sorte que, les coûts et les bénéfices qui découlent d'une participation équitable de la femme dans le développement devraient êtres considérées comme faisant partie du processus même de développement.

- Approche Empowerment et autonomie : L'apparition de cette approche remonte aussi aux années soixante, et s'oriente vers un renforcement et un élargissement de la base du pouvoir de la femme pour atteindre des degrés plus élevés d'autonomie citoyenne et d'autosuffisance. Elle encourage l'action collective et l'organisation et considèrent que les femmes doivent être capables d'agir avec une plus grande incidence sur leur environnement et avoir une participation active dans les risses de décisions qui affectent leur vie ou celles de la communauté, ce qui semble être primordial dans la lutte contre la pauvreté.

- Approche Droits: Les origines de cette approche remontent aux années quatre vingt dix et s'inscrivent dans les processus de globalisation. Il est question alors de changer la vision traditionnelle qui consiste à agir sur les besoins basiques, mais plutôt à s'orienter vers les droits fondamentaux de la femme. C'est un préalable pour un développement pour tous dans une perspective de droits humains. On ne considère plus la femme comme un acteur bénéficiaire passif mais plutôt comme agent productif actif ayant des droits d'accès dans des conditions d'égalité à ses bénéfices.

\section{RENFORCER L'ACQUISITION, L'INTEGRATION ET LE DEVELOPPEMENT DES DIFFERENTS SAVOIRS DE LA FEMME RURALE}

Les activités de formation doivent permettre, à différents niveaux de l'exercice des responsabilités au sein de l'organisation, l'acquisition et l'intégration de notions de gestion, de commercialisation, de production, ... ce qui permettra à chaque membre la pleine participation à la vie associative de leur coopérative, à l'exercice démocratique du pouvoir et donc assurer ainsi en quelque sorte la durabilité sociale et économique de la coopérative.

Permettre aux femmes d'accéder à une formation adaptée et continue devrait les aider à développer différentes habiletés entrepreunariales et associatives dans leur vie professionnelle comme dans leur vie familiale ou personnelle. C'est une façon de s'approprier le pouvoir dans leur lieu de travail et d'agir en solidarité avec les autres femmes et hommes de leur organisation. La formation sous toutes ses formes est primordiale pour la femme pour accéder à une pleine participation, à l'émergence des processus d'équité, d'empowerment et d'égalité des droits. 
Ci-après quelques exemples d'actions de formation orientés vers les femmes (simples salariées et/ou occupant un poste de responsabilité dans leur coopérative) et destinés à renforcer leurs capacités à accroître leur niveau de vie et leur pouvoir de décision sur ce qui les entoure:

1 Esprit d'initiative: encourager et initier les femmes à la création d'une coopérative (les étapes initiales de création et un plan d'action simple qui leur permettent de démarrer l'activité d'une manière efficace).

2 Le marché et la commercialisation: aider à appréhender les circuits de commercialisation des produits de la coopérative.

3 Calcul des prix et sélection des produits: accroître les compétences des membres d'une coopérative à évaluer la capacité de production, fixer les prix optimum et choisir les meilleures opportunités de travail pour la coopérative.

4 Gestion financière : permettre aux membres de la coopérative de bien gérer leurs finances et apprendre à préparer et à utiliser des états financiers.

5 Sources de financement: informer les membres de la coopérative sur l'identification et les sources de financement appropriées.

6 Organisation et décision: apprendre à établir des plans pour une meilleure prise de décisions.

7 Diagnostique et solutions : pouvoir identifier les raisons de l'échec et si possible trouver les solutions appropriées.

8 Planification pour le développement: apprendre à planifier et à budgétiser des actions ou des projets futurs.

\section{UNE PLUS GRANDE PARTICIPATION DE LA FEMME RURALE AU DEVELOPPEMENT SOCIAL, ECONOMIQUE ET PRODUCTIF}

La fonction reproductrice de la femme continue à être stigmatisée dans le marché du travail. Ceci a conduit les entreprises à préférer d'embaucher les hommes pour des postes permanents et mieux payés, alors que les femmes occupent des postes généralement d'ouvrières non qualifiées. D'autre part, la traditionnelle (supposée) dépendance de l'homme comme source de revenus a obligé la femme à dépendre de l'autorisation du mari pour assumer de nouvelles fonctions. Ces modèles culturels l'empêchèrent de développer l'image de femme citoyenne et agent du changement.

La diminution progressive de l'offre d'emploi dans le secteur public, conséquence directe des programmes d'ajustement structurel, a contraint hommes et femmes à s'orienter vers le secteur privé. C'est dans ce dernier secteur que les hommes 
jouissent de meilleures offres d'emploi, de meilleurs salaires, alors que la femme y trouve difficilement un emploi en grande partie à cause de ses responsabilités familiales et, de sa fonction de reproduction perçue comme source de complications pour l'employeur potentiel. A cela il faut ajouter, comme nous l'avons signalé précédemment, les faibles niveaux d'éducation, une moindre qualification, des barrières sociales et culturelles, tous ces facteurs réduisent les chances de la femme à trouver un emploi de salarié, ou si elle le trouve, c'est généralement avec une rémunération plus faible.

Ceci obligea les femmes, afin de garantir leur subsistance et celles de leurs enfants, à chercher du travail comme domestiques, ou à travailler pour leur propre compte, c'est l'auto emploi, ou dans le cadre essentiellement de la micro entreprise.

Les trois principales caractéristiques de ces micros entreprises sont :

a) On y développe des activités de production ou de vente de biens et services avec des niveaux de capitalisation très bas et un accès très limité aux marchés.

b) Trouvent leur origine dans la situation de précarité (faute d'emploi) et les besoins de survie.

c) Elles opèrent généralement dans des secteurs non structurés ou secteurs informels, parfois même dans l'illégalité.

Ces caractéristiques indiquent que l'auto emploi est "l'anti chambre » de la micro entreprise. Ainsi, l'auto emploi, en permettant à la femme d'exercer des activités productives et, dans la mesure où il contribue à l'emploi et à la génération de revenus (en espèces ou en nature) représente une manière de commencer à rendre plus visible la participation de la femme dans l'économie nationale.

\begin{tabular}{|c|c|c|}
\hline \multicolumn{3}{|c|}{ Classification des femmes selon les activités pour leur propre compte } \\
\hline STATUT & DESCRIPTION & $\begin{array}{c}\text { NIVEAU } \\
\text { D'ACTIVITE }\end{array}$ \\
\hline $\begin{array}{l}\text { Auto emploi pour } \\
\text { l'auto consommation }\end{array}$ & $\begin{array}{l}\text { Production de produits du secteur primaire (agriculture, pêche, } \\
\text { artisanat,...) pour une auto consommation }\end{array}$ & Subsistance $^{\mathrm{a}}$ \\
\hline $\begin{array}{l}\text { Auto emploi } \\
\text { marginal }\end{array}$ & $\begin{array}{l}\text { Activités de production ou de ventes de biens et services comme } \\
\text { l'élaboration de repas, confection d'habits, lavage et repassage de } \\
\text { vêtements d'autrui, ... }\end{array}$ & Subsistance \\
\hline Micro entreprise & $\begin{array}{l}\text { Activités de production ou de ventes de biens et services avec } \\
\text { utilisation d'une main d'oeuvre salariée ( } 1 \text { à } 4 \text { ou } 1 \text { à } 10 \text {, selon le cas) }\end{array}$ & $\begin{array}{l}\text { Accumulation } \\
\text { simple }^{\mathrm{b}}\end{array}$ \\
\hline Petite entreprise & $\begin{array}{l}\text { Activités de production ou de ventes de biens et services avec } \\
\text { utilisation d'une main d'oeuvre salariée (de } 10 \text { à } 50 \text {, selon le cas). }\end{array}$ & $\begin{array}{l}\text { Accumulation } \\
\text { large }^{c}\end{array}$ \\
\hline $\begin{array}{l}\text { a. Subsistance: Activ } \\
\text { de travail, permettent } \\
\text { b. Accumulation simp } \\
\text { Permet à p } \\
\text { c. Accumulation large } \\
\text { Les activités permett }\end{array}$ & $\begin{array}{l}\text { ités productives qui ne rémunèrent pas pour la capitalisation et ne couvi } \\
\text { peine de subsister. } \\
\text { le : } \\
\text { eine de couvrir les coûts directs du processus productif. } \\
\text { nt de couvrir tous les coûts, rémunèrent le propriétaire et génèrent des }\end{array}$ & éfices. \\
\hline
\end{tabular}

BARATARIA. Revista Castellano-Manchega de Ciencias Sociales No 9, pp. 63-81, 2008, ISSN: 1575-0825 
Cette stratification (au sens des critères du BIT), doit nous permettre de dessiner des stratégies et des politiques en adéquation avec le niveau dans lequel se trouve la population sur laquelle nous souhaitons agir. Par exemple, l'acquisition de compétences se transforme en une ressource importante qui permettra aux femmes de progresser de l'auto emploi à l'auto emploi marginal et de celui-ci à la micro entreprise.

Faute d'autres moyens de financement, le crédit peut s'avérer une ressource importante pour passer de l'auto emploi marginal à la micro entreprise et ensuite à la petite entreprise.

Certaines caractéristiques des micros entreprises gérées par des femmes, selon Josiane Capt (BIT, 1998), se présentent comme suit :

- Les micros entreprises sont créées avec les «moyens de bord», souvent sans une formation adéquate.

- Les micros entreprises se développent dans la sphère domestique, en général autour d'activités traditionnellement, féminines et avec des capitaux et des ressources limités.

La grande majorité des micros entreprises n'est pas enregistrée et opéré dans le secteur non structuré de l'économie. Dans la majorité des cas, elles réalisent leurs activités de production où offrent leurs services dans le foyer, dans les mêmes endroits où se développent les activités domestiques. Elles dépendent en général des travailleurs issus de la famille (rémunérés ou non).

Elles se concentrent dans des secteurs où il y une grande concurrence car c'est ceux qu'elles connaissent le mieux (vêtements, artisanat, pâtisseries, produits agroalimentaires,...).

La charge de travail est en général considérable, puisqu' à l'activité économique s'ajoutent les tâches domestiques. Il n'existe pas une división claire entre les « affaires » et le foyer.

La propriétaire de la micro entreprise exécute, en général, elle-même toutes les fonctions. La commercialisation et la gestion y sont rudimentaires et peu différenciées.

La production se commercialise localement ou est destinée aux marchés du tourisme et rarement à l'exportation (en particulier les produits artisanaux).

Trop dépendante de l'entourage socio culturel et des services de transport, les femmes peuvent commercialiser directement la production ou recourir à des intermédiaires masculins.

Les femmes rurales, quant à elles, sont en plus victimes de la discrimination historique faite par les pays en voie de développement entre le rural et l'urbain, en découle pour la femme rurale un accès plus faible à la formation et à tout type de services que pour la femme urbaine. Son intégration au monde du travail rémunéré est plus 


\section{Laila Hilal}

difficile, ce qui provoque un arrêt dans son développement et sa formation faute de moyens.

Dans le monde rural, les contributions de la femme à l'économie et au développement, sont encore moins visibles. Quand elles réalisent des travaux productifs à l'extérieur du foyer (cour de la propriété ou dans les champs avoisinants), ceux-ci ne sont pas considérés comme faisant partie d'un processus productif mais plutôt comme le prolongement de son rôle reproductif.

De plus, les statistiques nationales dépourvues de toute approche genre ne traduisent pas les apports de la femme rurale à l'économie familiale et au développement, les qualifiant d'inactives économiquement et dénommées « femmes au foyer ». Cette carence d'informations ne contribue pas au développement de stratégies d'action pertinentes et adaptées à la réalité de la campagne de la part des décideurs dans les politiques nationales de développement des secteurs tels que l'agroalimentaire, la santé, l'éducation et le commerce.

Les données de la FAO indiquent que dans les pays en développement, dont le Maroc, il y a une augmentation constante de la proportion de femmes dans la force de travail agricole par rapport à l'homme (phénomène appelé «féminisation de l'agriculture »). Quelques uns des facteurs qui expliquent une présence de plus en plus élevée des femmes dans la force de travail agricole sont :

a) L'émigration masculine à la recherche d'opportunités de travail dans les zones urbaines voire même à l'étranger (immigration), laissant l'agriculture entre les mains des femmes, enfants et grands parents.

b) Une amélioration dans le recueil de données statistiques nationales, dans le secteur informel, là où exercent la majorité des femmes.

c) La femme tend à occuper plus fréquemment des postes de faible productivité, particulièrement dans le secteur agricole.

Les stratégies d'action doivent rendre visible le travail de la femme rurale, améliorer son niveau de vie et celui de sa famille et lui permettre une pleine participation aux prises de décisions. Pour ce faire, il faut rompre les obstacles qui empêchent une intégration effective de la femme dans le développement économique et social, comme par exemple:

- Son apport à l'économie et à la société est historiquement rendu visible.

- Il existe un ensemble de normes sociales et culturelles qui constituent une barrière au développement.

- Les fonctions reproductives entièrement à sa charge ont limité sa participation dans la sphère productive. 
Evolution de la population active agricole

\begin{tabular}{|c|c|c|c|c|c|}
\hline & 1950 & 1970 & 1990 & $\begin{array}{c}\mathbf{2 0 0 0} \\
\text { (estimation) }\end{array}$ & $\begin{array}{c}\mathbf{2 0 1 0} \\
\text { (estimation) }\end{array}$ \\
\hline 1. Population agricole, dans le monde (en milliers d'hab.) & $1,621,927$ & $2,001,885$ & $2,465,015$ & $2,601,722$ & $2,667,219$ \\
\hline $\begin{array}{l}\text { 2. Part de la population agricole des pays en développement } \\
\text { dans le monde (\%) }\end{array}$ & 81.39 & 90.28 & 94.50 & 96.09 & 97.12 \\
\hline $\begin{array}{l}\text { 3. Part de la main d'oeuvre agricole féminine des pays en } \\
\text { développement dans le monde (\%) }\end{array}$ & 68.40 & 69.09 & 73.53 & 75.83 & 78.29 \\
\hline $\begin{array}{l}\text { 4. Main d'oeuvre féminine agricole dans le monde (en milliers } \\
\text { de pers.) }\end{array}$ & 315,924 & 382,605 & 523,315 & 579,771 & 621,308 \\
\hline $\begin{array}{l}\text { 5. Part de la main d'oeuvre féminine des pays en } \\
\text { développement dans le monde }\end{array}$ & 82.17 & 89.86 & 95.43 & 97.01 & 97.98 \\
\hline \multicolumn{6}{|l|}{$\begin{array}{l}\text { 6. Part de la main d'oeuvre féminine agricole dans la population } \\
\text { agricole active totale }(\%)\end{array}$} \\
\hline 6.1 Monde & 39.01 & 41.13 & 42.69 & 43.55 & $44.16>$ \\
\hline 6.2 Pays développés & 41.07 & 43.77 & 38.38 & 36.13 & 33.73 \\
\hline 6.3 Pays en développement & 38.59 & 40.85 & 42.93 & 43.83 & 44.44 \\
\hline $\begin{array}{l}\text { 7. Part de la main d'oeuvre agricole dans la main d'oeuvre totale } \\
(\%)\end{array}$ & 89.56 & 84.21 & 75.97 & 70.62 & 65.21 \\
\hline $\begin{array}{l}\text { 8. Part de la main d'oeuvre féminine dans la population agricole } \\
\text { active totale }(\%)\end{array}$ & 44.70 & 45.87 & 47.34 & 48.10 & 48.74 \\
\hline
\end{tabular}

\section{RENFORCER L'EMPOWERMENT DES FEMMES RURALES}

L'expérience récente a montré que beaucoup de projets de développement axé sur le genre et financés par des institutions nationales, ONGs, coopératives ou organismes internationaux, n'atteignent leurs objectifs : réduire considérablement la pauvreté féminine. Nous croyons que l'une des principales raisons est que pour ces institutions souvent, il semble plus primordial d'assurer une durabilité financière au projet que de se pencher sur les vraies raisons d'une paupérisation résistante de la femme en général et, rurale en particulier.

En effet, l'empowerment (ou renforcement des capacités) de la femme, dans un BARATARIA. Revista Castellano-Manchega de Ciencias Sociales № 9, pp. 63-81, 2008, ISSN: 1575-0825 
sens économique, social et politique n'est pas perçu comme une priorité dans les stratégies d'action dans la lutte contre la précarité dans laquelle se trouve la femme rurale. Or, si les femmes n'atteignent pas un niveau plus élevé d'empowerment social et politique, quelque soit le programme de développement dirigé vers elles, il ne pourra soulager la pauvreté pour toujours car il n'aura pas servi pour la rendre plus capable de se prendre en charge et de décider de son propre destin, d'avoir un pouvoir et de travailler à ses intérêts stratégiques en plus de ses besoins pratiques. Pour atteindre l'égalité entre les genres, il est donc indispensable que les femmes s'approprient le pouvoir sur leur force de travail et sur leur destinée en général.

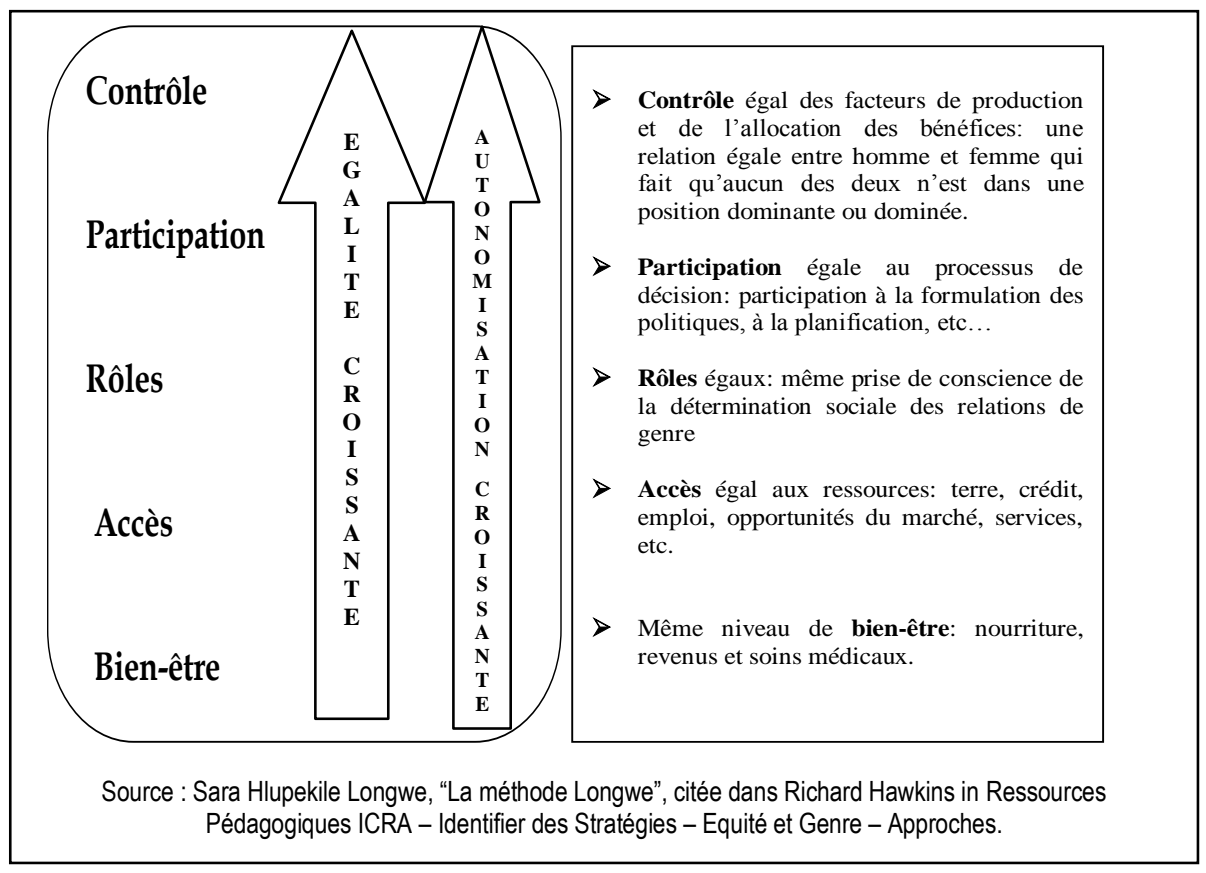

Linda Mayoux a écrit: “ $L$ 'empowerment en soi contribue considérablement à la durabilité financière. L'empowerment économique (plus de revenus, un contrôle sur ces revenus, un accès aux ressources) réduit les risques d'échec. Plus de bien être signifie que les femmes ont plus de connaissances, de temps, une possibilité plus grande d'acquérir d'autres ressources pour contribuer à la réussite des programmes de développement. L'empowerment social et politique augmente l'empowerment 
économique et le bien être, en permettant aux femmes de participer plus activement aux programmes en raison d'une plus grande liberté de mouvements et de contact avec les hommes et, une plus grande capacité d'organisation et de travail en réseaux.

\section{EN GUISE DE CONCLUSION}

Le thème de la présente recherche est «l'implication et la participation des femmes dans le développement et dans la construction de leur identité ».

L'objectif général de notre approche est d'analyser les implications de la participation de la femme en milieu du travail sur le processus de la reconstruction de son identité de genre.

Pour les hommes qui côtoient ces femmes dans le travail, dans le foyer, dans la rue, se posent trois grandes catégories d'analyse :

1. Construction de l'identité genre;

2. Travail féminin et son incidence sur la reconstruction de l'identité de genre ;

3. Participation au milieu laboral et son incidence sur la reconstruction de l'identité de genre.

Cette recherche est avant tout qualitative, réalisée par la collecte de données. Les données ont été collectées à partir d'observation participative, de récits de vie et d'entretiens semi directifs.

Les résultats obtenus ont montrés que les femmes construisent leur identité à partir de rôles traditionnellement définis, dans lesquels la femme a la responsabilité de la reproduction familiale (transmission des valeurs, éducation des enfants, travaux domestiques, reproduction de la force de travail). Ceci donne une division sociale qui se répercute sur les espaces tant en milieu de travail et social que familial, où traditionnellement la femme occupait l'espace privé et domestique et l'homme l'espace publique.

Dans la deuxième phase de l'analyse, nous avons observé que le travail productif génère des changements importants dans la vie de la femme puisque, une fois qu'elle peut s'investir dans d'autres espaces et acquérir un salaire en contrepartie de leur travail, elle commence à développer une autonomie qu'elle n'avait pas antérieurement. Elle a alors la possibilité de gérer ses revenus et ne dépend plus uniquement des revenus masculins pour subvenir à ses besoins.

Finalement, les femmes qui participent dans diverses associations et coopératives y voient une opportunité pour occuper les espaces jusque-là interdits et induire un processus d'identification entre elles-mêmes, qui peut à son tour produire une amélioration de leur condition de travail et l'élaboration de proposition en tant que collectif.

Actuellement les bénéfices de cette participation s'observent au niveau 


\section{individuel, comme la possibilité d'avoir des postes de responsabilité au sein de l'organisation et l'accès aux divers avantages comme le micro crédit, la formation.}

\section{NOTES}

${ }^{1}$ Préparation d'un Doctorat National sous la Direction du Pr. Rachid BELHAJ sur le thème «Communication sociale en milieu rural. Intervention systémique au bénéfice de la femme en milieu rural : Impact du Travail sur l'Identité de Genre ».

${ }^{2}$ Dans le cadre associatif ou dans nos recherches dans le cadre du doctorat.

${ }^{3}$ Présidente de la Section de Boukhalef-Tanger de l'Union Nationale des Femmes Marocaines depuis 1998, membre fondateur de l'association DARNA-Tanger (1995) et de AFEM (Association des Femmes Chefs d' Entreprises Marocaines, Section Tanger, 2000) et membre active de plusieurs associations tangéroises qui oeuvrent pour l'amélioration des conditions de vie des groupes de population défavorisée.

${ }^{4}$ La Déclaration et la Plateforme d'Action (PdA) de Beijing de 1995, le rapport sur le Développement Humain du PNUD de 1995 et postérieurement, en 2000, les Objectifs de Développement du Millénaire (ODM).

${ }^{5}$ Coûts d'efficience: car cette politique favorise les activités des hommes qui ne sont pas nécessairement les plus efficientes; coûts de bien-être: dû à ce que cette politique implique une redistribution des ressources entre hommes et femmes qui privilégient les premiers; coûts d'équité: car cette politique réduit la capacité productive indépendante et l'autonomie économique des femmes. ${ }^{6}$ La croyance populaire.

${ }^{7}$ In Durstewitz P. (2000), « La perspective de genre dans les micros finances ».

${ }^{8}$ Rapport 2007 du BIT sur « Les tendances mondiales de l'emploi des femmes ».

${ }^{9}$ Ministère de l'Economie et des Finances, Direction des Etudes et des Prévisions Financières, « Impacts du Programme d'Ajustement Structurel », 1996, document de travail numéro 13.

${ }^{10}$ Moser (in Williams et al, 1995) décrit cette évolution en faisant la distinction entre plusieurs cadres d'analyse des relations de genre.

11 Harper, Malcolm (1998), "Une coopérative de travailleurs: Formation et gestion: Guide de l'instructeur: matériau pour les assesseurs des coopératives", BIT, Genève.

${ }^{12}$ Définit comme l'exercice d'activités pour son propre compte sans utilisation d'aucune autre main d'œuvre alors que la micro entreprise se caractérise par l'emploi d'une main d'œuvre salariée régulière.

${ }^{13}$ In La vie économique du 31/12/04: La micro entreprise, Championne de l'économie, «Selon le Haut commissariat au Plan, 97,7\% du tissu économique marocain est constitué de micro-unités employant moins de dix personnes» (recencement économique 2001-2002).

${ }^{14}$ Rajaa MEJJATI ALAMI (2006), «Le secteur informel au Maroc : 1956 - 2004», GT3-7 418.

${ }^{15}$ Josiane Capt (1998), “Lesfemmes et le développement des micros entreprises”, BIT - 28 septembre 1998.

${ }^{16}$ Collection FAO: Dèveloppement statistique no. 9 (Report on the 1990 World Census of Agriculture, international comparison and primary results by country (1986-1995)), FAO, Rome 1997 http://www.fao.org/Gender/fr/stats-f.htm\#Maps

17 Population agricole (en milliers): Population totale: 31 064; Population agricole: 10 408; Population non agricole: 20656 - Source: 2004, FAOSTAT.

${ }^{18}$ Soiurce : Division de la statistique du Département économique et social de la FAO.

${ }^{19}$ Caubergs, L. (2002) : « Genre et Empowerment », in 
http://www.genreenaction.net/spip.php?article3237

${ }^{20}$ Mayoux L. (1998) : «L'empowerment des femmes contre la viabilité ? Vers un nouveau paradigme dans les programmes de microcrédit ». In : Preiswerk, Y., dir. - Les silences pudiques de l'économie, Économie et rapports sociaux entre hommes et femmes. Textes réunis par Commission nationale suisse pour l'Unesco, Direction du développement et de la coopération. Genève : Institut Universitaire d'Études du Développement.

\section{BIBLIOGRAPHIE}

ACHY, L.; DE HENAU, J.; EL KADIRI, N. ; KATEB, K.; LAUFER, C.; MARUANI, M.; MEULDERS, D.; SILVERA, R. ; SOFER, C. y ZOUARI, S. (2004): Marché du travail et genre, Maghreb-Europe, Brussels Economic Series, Bruxelles, Editions DULBEA.

ALAMI M'CHICHI, H. (2002): Genre et politique au Maroc. Les enjeux de l'égalité hommes-femmes entre islamisme et modernisme, Paris, L'Harmattan.

ALBARELLO, A. (1995): Pratiques et méthodes de recherche en sciences sociales, Paris, Armand Collin. AMAPPE (2000): Annuaire des associations marocaines de développement », ODCO et Banque Mondiale, Rabat, Editions OKAD.

AMARA, F. (2003): Ni putes, ni soumises, Paris, La Découverte, p. 88-89.

AMELLAL, K. (2005): Discriminez-moi! Enquête sur nos inégalités, Paris, Flammarion.

ARENDT, H. ; CLAUDEL, C. ; DOLTO, F. ; ROOSEVELT, E. y SCHUMANN, C. (2006): L'Universel au Féminin, Paris, L’Harmattan.

LAROUSSI, A. (2003):La femme rurale dans l'exploitation familiale nord-ouest de la Tunisie, Pour une sociologie des ruptures, Paris, L'Harmattan.

ASSOCIATION DEMOCRATIQUE DES FEMMES DU MAROC (2001): Les discriminations à l'égard des femmes dans la législation marocain, Casablanca, Editions Le Fennec.

BANQUE MONDIALE (2004): Mondialisation, développement et pauvreté; bâtir une économie intégrée, Paris, Editions Eska.

BARD, C. ; BAUDELOT, C. y MOSSUZ-LAVAU, J. (dir.) (2004): Quand les femmes s'en mêlent, Paris, Editions de la Martinière.

BARKALLIL N. (1997): La question féminine au Maroc, occident de l'Islam, in BISILLIAT, J. (dir.), Face au changement, les femmes du sud, Paris, L'Harmattan.

BEAUVOIR (de), S. (1950): Le deuxième sexe, Paris, Gallimard, t.1: Les faits et les mythes, t.2 :L'expérience vécue.

BELARBI, A. ; AMITI, F. y BENARAFA, C. (1990): Etre jeune fille, Casablanca, Editions Le Fennec.

BELARBI, A. (dir.) (1999) : «Initiatives féminines », Casablanca, Editions Le Fennec.

BENCHERIFA, A. y SWEARINGEN, W. D. (ed.) (1995): L'Afrique du nord face aux menaces écologiques, Rabat, Publications de la Faculté des Lettres et des Sciences Humaines, Univ. Mohamed V.

BENNANI-CHRAIBI, M. ; FILLIEULE, O. (2003) : «Résistances et protestation dans les sociétés musulmanes », Paris, Oresse de Sciences Po.

BEN NEFISSA, S. (2002): «Pouvoirs et associations dans le monde arabe», Paris, Editions CNRS.

BERTAUX, D. (2005): «L'enquête et ses méthodes : Le récit de vie », Armand Colin,.

BESSETTE, G. (2004): «La communication participative pour le développement : un agenda ouestafricain », Editions Centre de recherches pour le développement international (CRDI).

BESSIS, S. (1996): «La féminisation de la Pauvreté », in BISILLIAT, J, (dir.), Femmes du sud, chefs de famille, Paris, Karthala.

BARATARIA. Revista Castellano-Manchega de Ciencias Sociales № 9, pp. 63-81, 2008, ISSN: 1575-0825 
BOURDIEU, P. (1998): «La domination masculine », Paris, Seuil.

CARRYER, B. (2005): «Femmes rurales dans le Mozambique contemporain. Politique et quotidien, une émancipation manquée? », Paris, Éditions L'Harmattan, Collection Alternatives rurales.

CASPAR, R. (1990): «Pour un regard chrétien sur l'Islam », Paris, Centurion.

CHAFIQ, C. (1991): «La femme et le retour de l'Islam», l'expérience iranienne, Iran, Felin Editions.

DAOUD, Z. (2004): «Marocains de l'autre rive : Les immigrés marocains, acteurs du développement durable», Paris, Editeur Paris-Méditerranée.

DE SEQUERIA CARVALHO, J. A. (2004): «La Dynamisation des initiatives locales», Paris, Éditions L'Harmattan.

DOUCET, L. Y FAVREAU, L. (1991): Théorie et pratiques en organisation communautaire, Presses de l'Université du Québec.

DUPUY, F. (2001): L'alchimie du changement. Problématique, étapes et mise en æuvre, Paris, Dunod.

DURET, P. (1999): Les jeunes et l'identité masculine, PUF.

DURU-BELLAT, M.( 1989): L'école des filles. Quelle formation pour quels rôles sociaux?, Paris, L'Harmattan.

EARL, S.; CARDEN, F. y SMUTYLO, T. (2002): La cartographie des incidences, Intégrer l'apprentissage et la réflexion dans les programmes de développement, Montréal.

EDUARDS, M. (1995): La place des femmes. Les enjeux de l'identité et de l'égalité au regard des sciences sociales, Paris, Recherches EPHESIA, La Découverte.

ENGELS, F. (1984): «Les origines de la famille, de la propriété privée et de l'Etat», Version téléchargeable http://classiques.uqac.ca/classiques/Engels_friedrich/Origine_famille/Origine_famille.html FALCONNET, G. y LEFAUCHEUR, N. (1975): La fabrication des mâles, Paris, Seuil.

FEDIDA, P. y UYOTAT, J. (dir.) (1986): Mémoires, Transferts, Echo-Centurion,.

FILALI MEKNASSI, R. (2001): Analyse de la situation des femmes et des enfants au Maroc, Rabat, AMS/UNICEF. FORTINO, S. (2002): La mixité au travail, Paris, Editions La Dispute/SNEDIT.

GALLAND, C. (1995): Femmes du sud: Sources d'information pour le développement, Paris, Ibiscus.

GARANT, M. y SCIEUR P. (dir.) (2002): Organisations et systèmes de formation, Bruxelles, De Boeck.

GRENIER, L. (1998): Connaissances indigènes et recherche, Un guide à l'intention des chercheurs, Editions Centre de recherches pour le développement international (CRDI),.

KABEER, N. (2005): Intégration de la dimension genre à la lutte contre la pauvreté et objectifs du millénaire pour le développement, Manuel à l'intention des instances de décision et d'intervention, Laval, Les Presses de l'Université Laval.

KUNDERA, M. y FRANÇOIS R. (2000): L'Identité, Gallimard.

LAUFER, J.; MARRY, C. y MARUANI, M. (dir.) (2003): Le travail du genre. Les sciences sociales du travail à l'épreuve des différences de sexe, Paris, La Découverte-MAGE.

LE DOEUFF, M. (1998): Le sexe du savoir, Paris, Editions Alto-Aubier.

LE MOIGNE, J.-L. (1996): Les épistémologies constructives, PUF, Collection Que sais-je?

LEZINE, I. (1965): Le développement psychologique de la première enfance, Paris, PUF.

LUSTHAUS, C. ; ADRIEN, M.-H. ; ANDERSON, G. y CARDEN, F. (1999): Améliorer la performance organisationnelle, Manuel d'autoévaluation, Montréal, Editions Centre de recherches pour le développement international (CRDI).

MANTRAN, R. (1995): L'Expansion musulmane, VIIe-XIe siècles, Paris, PUF, 6éd.

MARUANI, M. (dir.) (2005): Femmes, genre et sociétés. L'état des savoirs, Paris, La Découverte.

MARUANI, M. (,2000): Travail et emploi des femmes, Paris, Edition La Découverte, Collection Repères.

MEAD, G. H. (2006): L'esprit, le soi et la société, Paris, PUF, Collection Lien Social.

MEILLASSOUX, C. (1977): Femmes, greniers, capitaux, Paris, Maspéro.

MELEN, D. (2001): Luttes contre la pauvreté en milieu rural, Laval, Les Presses de l'Université Laval.

BARATARIA. Revista Castellano-Manchega de Ciencias Sociales No 9, pp. 63-81, 2008, ISSN: 1575-0825 
MERNISSI, F. (1999): Le harem politique, Paris, Complexe.

MERNISSI, F. (1983): Le Maroc raconté par ses femmes, Rabat, SMER.

MICHEL, A. (2003): Le féminisme, Paris, Presses Universitaires de France - PUF; $7^{\text {ème }}$ Édition, Collection Que sais-je, N 17820.

MILLETT, K. (1971): La politique du mâle, Paris, Stock.

MOHA, E. (1995): Histoire des relations franco-marocaines, ou les aléas d'une amitié, Jean PICOLLEC. MUCCHIELLI, A. (1992): L'identité, Paris, PUF.

NAAMANE-GUESSOUS, S. (1991): Au delà de toute pudeur : la sexualité féminine au Maroc, $7 \mathrm{e}$ éd. rev. et corrigée, Casablanca, Éd. Eddif.

PASCON, P. (1980): Etudes rurales, idées et enquêtes sur la campagne marocaine, in- $8^{\circ}$ br., Rabat, Société Marocaine des Editeurs Réunis.

PERRENOUD, P. (2004): Desarrollar la práctica reflexiva, Barcelona, Grao.

PERROT, M. (2001): Les Femmes ou les silences de l'histoire, Paris, Flammarion.

PLANTADE, N. (1988): La guerre des femmes, Paris, La boîte à Documents.

PREFOL, P. (1986): Prodige de l'irrigation au Maroc. Le développement exemplaire du Tadla 1936-1985, Paris, Nouv. Ed. Latines, in $-8^{\circ}$ br.

ROSANDER, E. (1999): Transformation des identités féminines : formes d'organisation féminines en Afrique de l'Ouest, Stockholm et Uppsala, Gotab and Nordiska Afrikainstitutet.

SAADI, N. (1991): La Femme et la Loi en Algérie, Casablanca, Edition Le Fennec.

SAHER, S. (2005): Impact les ONG ont-elles sur les discours et les situations des femmes, Paris, Editions Univ.

THEDE, N. (2000): Gitans et flamenco, les rythmes de l'identité, Paris, L'Harmattan.

TOZY, M. (1999): Monarchie et islam politique au Maroc, Paris, Presses de Sciences Po.

VIAU, R. (2000): Femmes de personne, Montréal, Ed. Boréal.

ZOUGGARI, A. (1996): L'école en milieu rural, Rabat, Fondation Konrad Adenauer.

\section{Breve currículo :}

\section{Laila Hilal}

Profesora Titular de comunicación en el Instituto Internacional Superior de Turismo de Tánger. Presidenta de la asociación Unión de mujeres marroquíes Sección de Boukhalef, Tánger. Trabajos recientes: Globalización e interculturalidad en la empresa, (2006); Genero y desarrollo, (2006); "La mujer en un país en movimiento", (2004); "Mujeres y cambios sociales en Marruecos", Igualdad, participación y Valores Sociales entre el hoy y mañana, (2005); «La Mudawana dos años después: Balance y perspectivas», Seminario Hispano-marroquí sobre la situación jurídica de la mujer , Universidad de Cádiz, 2005; Iniciadora y responsable del proyecto ADER con el apoyo de la Fundación Bernard Van Leer, La Haya, 2004: "Ayuda al desarrollo de la pequeña infancia y la familia en el medio rural"; "Mujeres y cambios sociales en Marruecos", Igualdad, participación y Valores Sociales entre el hoy y mañana, Sevilla, 2005. 